\title{
Un exemple d'interaction fluide-fluide : « essorage par un jet d'air turbulent d'un film liquide entraîné par une plaque en mouvement
}

\author{
Éric Arquis ${ }^{1, a}$, Delphine Lacanette $^{1}$, Stéphane Vincent ${ }^{1}$, Jean-Paul Caltagirone ${ }^{1}$ \\ ET PASCAL GARDIN ${ }^{2}$ \\ 1 Laboratoire Inter-établissements TREFLE - UMR CNRS 8508, Site ENSCPB, 16 Av. Pey-Berland, \\ 33607 Pessac Cedex, France \\ 2 ARCELOR Research, Voie Romaine, 57280 Maizières-lès-Metz, France
}

Reçu le $1^{\text {er }}$ septembre 2006, accepté le 23 janvier 2007

\begin{abstract}
Résumé - Cet article présente une simulation numérique des interactions hydrodynamiques entre une plaque solide en mouvement, un film liquide entraîné par cette plaque et un fluide gazeux au repos ou structuré en jet plan impactant ce film, dans une hypothèse de géométrie cartésienne $2 \mathrm{D}$ essentiellement, mais également 3D. Cette problématique se rencontre dans des applications procédés « matériaux » (contrôle d'épaisseur de dépôt ou d'états de surface en sidérurgie) ou pour dans les parois protectrices (des véhicules spatiaux en phase de rentrée atmosphérique ou des réacteurs de fusion nucléaire) et d'une certaine façon dans des problèmes de condensation avec balayage par un écoulement gazeux. Ce problème allie de nombreuses difficultés physiques et numériques : traitement des interfaces liquide-gaz et des instabilités qui y prennent naissance, turbulence dans la zone gazeuse et éventuellement dans la zone liquide. Une autre difficulté est inhérente aux multiples échelles rencontrées : procédé à l'échelle centimétrique-métrique, épaisseur de film à l'échelle millimétrique-micronique, échelles de turbulence variées. Notre travail va tenter de vaincre ces difficultés et de produire des résultats en conditions réelles ... ou du moins au plus près de celles-ci.
\end{abstract}

Mots clés : Film entraîné / interaction liquide-gaz / jet plan turbulent / LES

Abstract - Example of fluid-fluid interaction: air-jet wiping of a liquid thin film dragged by a moving plate. This works deals with the numerical simulation of hydro-dynamical interactions between a solid moving plate, a metallic liquid film covering it and a gaseous fluid at rest or structured as an air-jet, mainly in the frame of 2D-Cartesian geometry but also in full 3D. This problematic is encountered in Material Processing applications (such as the controlling of thickness of the liquid film in metallurgy, the paper industry, covered fibre manufacturing) or for Thermal Protection Walls (spatial vehicles in atmospheric re-entry phase or in confinement walls for nuclear fusion reactors) and in some ways, it is also encountered in problems of condensation interacting with air-flows. This problem combines physical and numerical difficulties related to the treatment of liquid-gas interfaces, the appearance of front instabilities, stemming from the turbulent nature of the flow in the gas and eventually in the liquid itself. An additional challenge is inherent to the various time and space scales: the process is typically at a centimetre-metre scale whilst the film should be tracked at both millimetre and micron scale, and the turbulence induced by the jet flow covers a large spectrum of scales. The present work will try to overcome all these difficulties in order to produce results in real conditions with appropriate and manageable modelling.

Key words: Dragged film / liquid-gas interaction / plane turbulent jet / from tracking / LES modelling

\section{Introduction}

La présente étude fait référence à un problème couplé d'enduction-essorage. On rencontre des phénomènes

\footnotetext{
a Auteur correspondant : arquis@enscpb.fr
}

d'enduction dans de nombreuses applications industrielles : papier, fibres, produits métalliques. Le principe général est simple : un support, fil ou plaque, est tiré d'un bain d'un milieu visqueux, le déplacement entraînant un film liquide qui est ensuite figé par solidification ou 
réticulation selon le matériau. Outre aux phénomènes de mouillabilité, l'épaisseur finale du film, paramètre essentiel à contrôler dans le cadre industriel, est corrélée à la viscosité et à la masse volumique du fluide (cette dernière au travers des effets de gravité), à la vitesse de déplacement du support et dans une moindre mesure aux propriétés comparées du fluide entraîné (eau, métal, polymère, etc.) par rapport au fluide ambiant (rapport de viscosités, tension superficielle). Cette épaisseur «naturelle $»$, i.e. sous le seul effet du couplage entraînement visqueux/gravité est généralement trop importante : pour des raisons techniques et économiques on tente donc de trouver des solutions pour la réduire et surtout la contrôler. L'« essorage pneumatique » [1] est une de ces solutions : on fait impacter un jet sur le film qui, en subissant les efforts (pression, cisaillement) résultants, se trouve raboté. Encore faut-il pouvoir doser l'intensité du jet pour ne pas faire apparaître des phénomènes gênants sur l'installation industrielle telle que l'éclaboussement (« splashing » dans la terminologie anglaise).

Le problème du seul entraînement du film a fait l'objet d'une bibliographie large et relativement ancienne : on trouve notamment pour des configurations filaires ou planes des résultats analytiques [2]. Cependant ces résultats quoique fort intéressants et essentiels pour un premier dimensionnement du problème, sont basés sur des hypothèses (inertie négligée, interaction bain-film non prise en compte) qui sont critiquables par rapport aux conditions réelles.

Quant au problème de l'essorage, peu de travaux existent : là aussi on trouve des approches analytiques et expérimentales $[3,4]$, mais qui ne permettent pas de répondre à toutes les questions posées. Ce dernier problème nécessite en effet la prise en compte de façon la plus fidèle possible du couplage film-jet. Or pour que l'effet du jet soit significatif sur un film de fluide pesant et visqueux tel qu'un métal liquide, il faut atteindre des niveaux de contraintes que l'on ne peut obtenir, avec un gaz, qu'en régime turbulent.

La simulation numérique retenue est de type «semidirecte $»$ : en effet, si la simulation des phases séparées ne recourt pas à une modélisation des interactions, la turbulence quant à elle est modélisée dans le cadre d'une approche SGE (LES en anglais).

Cette simulation sera opérée en considérant un domaine incluant la plaque solide mobile, le fluide entraîné et le fluide ambiant au repos ou structuré sous forme de jet impactant : à noter que l'interface fluide 1 /fluide 2 sera déterminé par l'équilibre des forces en présence sans que ces forces soient explicitement formulées (écriture d'une équation de quantité de mouvement unique pour tous les fluides couplée à une méthode de suivi d'interface par équation d'advection).

Grâce à cet outil numérique, nous pouvons apprécier les effets à la fois géométriques (profondeur d'immersion de la plaque mobile, distance jet-plaque, etc.), opératoires (vitesses de plaque et de jet), des propriétés des fluides en présence (viscosités, masses volumiques) sur l'épaisseur, dite asymptotique, du film (i.e. en aval, loin

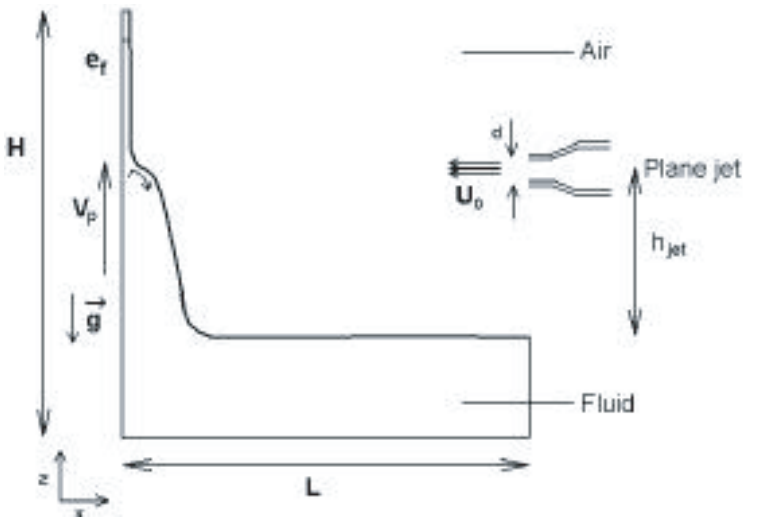

Fig. 1. Définition de la géométrie et notations géométriques.

de la zone d'impact du jet). Une analyse qualitative des phénomènes, complétée par des résultats quantitatifs est présentée : une comparaison avec des résultats théoriques, numériques et expérimentaux de la littérature ou sur installations industrielles sera notamment rapportée.

\section{Description du problème et méthodologie numérique}

La situation typique que nous souhaitons traiter est représentée sur la figure 1. Nous considérons une plaque (ou une bande) dont la largeur est suffisamment grande par rapport à son épaisseur pour que l'on puisse considérer le problème comme bidimensionnel, donc dans un plan vertical normal à la plaque. Cette hypothèse est géométriquement vérifiée, mais elle est critiquable vis-àvis du traitement de la turbulence que nous adopterons le plus souvent : au sens strict, la turbulence induite par un jet plan est 3D (certes faiblement). Afin de réduire le temps de calcul, une seconde hypothèse, de symétrie par rapport au plan vertical $x=0$, est faite : si là aussi elle est légitime dans la partie aérienne, elle est plus discutable dans le bain : en pratique la plaque est entraînée en partie basse par un rouleau et donc forcément l'écoulement n'y est pas symétrique.

Moyennant ces hypothèses, la plaque, ici sur la gauche du schéma, est figurée par une ligne de nœud unique sur laquelle est imposée la vitesse tangentielle, verticale donc $V_{z}=V_{\mathrm{p}}$, tandis qu'en dessous de la plaque dans la zone immergée, une condition de symétrie est posée $\left(\partial V_{z} / \partial x=0\right)$. La plaque est immergée dans un bain liquide de hauteur $h$ et d'extension latérale $L$ (cette extension correspondra par la suite à la distance buse du jet-plaque), tandis que la hauteur émergée qui correspond à la distance surface du bain-frontière supérieure du domaine de calcul est notée $H_{\text {air }}\left(H=H_{\text {air }}+h\right)$.

De manière à assurer une alimentation permanente du film, il convient de maintenir le bain à un niveau constant. Ceci pourrait être réalisé en assurant une alimentation permanente de ce bain par la zone droite exactement égale à la quantité de fluide sortant par entraînement en partie haute. Cette solution complexe a été abandonnée au profit 
d'une solution beaucoup plus simple qui consiste à imposer le niveau grâce à un traitement adéquat de l'équation de conservation de la fonction de présence du liquide (voir plus loin lors de la description des équations). Sur la partie basse du domaine, figurant le fond du bain une condition de paroi (adhérence et imperméable) est adoptée. Sur les faces droites et supérieures, une condition de type Neuman $\left(\partial V_{\text {normale }} / \partial n=0\right)$ est prise afin de permettre à la matière de rentrer... et de sortir. Cette condition s'applique sauf sur la zone correspondant au jet : le jet impactant émane d'une buse placée à une hauteur $h_{\text {jet }}$, la buse étant figurée comme une fente de largeur $d$. Un profil de vitesse uniforme d'intensité $V_{\text {jet }}$ est imposé sur toute la hauteur de la fente, mais d'autres profils peuvent être adoptés («top-hat » ou bien issus de simulations annexes de l'écoulement dans la buse, si disponibles).

Les fluides, que ce soit celui du bain ou celui de l'ambiance (et du jet puisqu'ils sont identiques) sont supposés newtoniens, incompressibles. On considérera ici pour le bain soit de l'eau, soit du zinc fondu, dont les viscosités cinématiques sont proches, mais de densités assez différentes (6 pour zinc/eau). Les gammes de vitesse du jet observées en pratique, $200 \mathrm{~m} . \mathrm{s}^{-1}$, font que l'hypothèse d'incompressibilité pour l'écoulement de jet est un peu discutable, car on atteint des nombres de Mach de 0,20,3 , mais nous avons pris le parti d'ignorer cette incidence. Pour la plaque, des vitesses de déplacement de 2 à $5 \mathrm{~m} . \mathrm{s}^{-1}$ seront considérées. La largeur de buse étant de l'ordre du millimètre, on voit que le nombre de Reynolds du jet est de l'ordre typiquement de 20000 . Quant à la distance buse-bande elle est de $10 \mathrm{~mm}$, d'où des rapports $L / d$ de l'ordre de 10 .

Dans le travail présenté ici, les aspects thermiques sont occultés : on prendra donc des caractéristiquesproduit (viscosité, masse volumique, tension superficielle) indépendantes de la température.

Moyennant toutes ces hypothèses, nous allons poser les équations régissant les phénomènes :

$$
\begin{aligned}
& \rho\left(\frac{\partial u}{\partial t}+(u \cdot \nabla) u\right)=\rho g-\nabla p \\
& +\nabla \cdot\left(\left(\mu+\mu_{\mathrm{T}}\right)\left(\nabla u+\nabla^{t} u\right)\right)+F_{\mathrm{TS}}-B_{\mathrm{u}}\left(u-u_{\infty}\right)
\end{aligned}
$$

$$
\begin{aligned}
& \nabla \cdot u=0 \\
& \frac{\partial C}{\partial t}+u \cdot \nabla C+B_{\mathrm{c}}\left(C-C_{\infty}\right)=0
\end{aligned}
$$

Par rapport à la forme classique de ces équations, il convient de donner quelques précisions :

- L'équation de Navier-Stokes (1) comme celle d'Advection (3) comportent un terme complémentaire qui permet de pénaliser grâce au paramètre $B$ la variable concernée, $u$ ou $C$, autrement dit de fixer la valeur de celle-ci à une valeur spécifiée $\left(u_{\infty}\right.$ ou $\left.C_{\infty}\right)$ : ceci permet de bloquer la vitesse dans les zones solides (plaque en mouvement) ou encore de fixer la couleur localisant un fluide donné (cf. maintien du niveau du bain mentionné plus haut)
- Il apparaît dans l'équation de Navier-Stokes un autre terme non conventionnel $F_{\text {TS }}$ formé avec le gradient de la fonction Couleur. Ce terme permet de tenir compte, par une écriture volumique compatible avec la stratégie de suivi d'interface choisie ici, des effets de tension superficielle. Cette technique proposée par Bradbill [5] et décrite dans [6] a été validée par Vincent et al. [7].

- L'équation de quantité de mouvement fait intervenir la viscosité turbulente car nous utilisons ici un modèle de type LES. Cette méthode de traitement de la turbulence a été retenue ici sous l'argument qu'avec un effort numérique raisonnable en termes de maillage et pas de temps, elle permet de rendre assez fidèlement des écoulements fortement cisaillés et instationnaires tels que rencontrés ici. Nous renvoyons le lecteur pour une description détaillée de la méthode à Sagaut [8]. De manière très lapidaire, nous dirons que la prise en compte des grandes échelles de la turbulence est rendue grâce à un modèle de sous-maille, modèle qui se traduit par la définition d'une viscosité turbulente, définie notamment par rapport à la taille des mailles (filtre spatial). Il existe de nombreuses variantes des modèles LES : nous avons retenu celle dit de Smagorinski où

$$
\mu_{\mathrm{T}}=\rho\left(C_{\mathrm{s}} \bar{\Delta}\right)^{2} \cdot \sqrt{2(\nabla u \otimes \nabla u)} \text { où } \bar{\Delta}=(\Delta x \Delta y \Delta z)^{1 / 3}
$$

$(\Delta x, \Delta y, \Delta z$ sont les tailles des mailles dans les directions $x, y$ et $z), C_{\mathrm{s}}$ étant pris égal à 0,18 dans le cadre d'une turbulence homogène isotrope. Pour les simulations 2D qui constituent l'essentiel de notre article, la dimension de maille $\Delta y$ n'existant pas, nous avons arbitrairement pris la définition suivante pour $\bar{\Delta}=\operatorname{Min}(\Delta x, \Delta z)$. Cette valeur a été calée sur les études de jet impactant sur plaque sèche et immobile par comparaison avec la littérature et a donné les meilleurs résultats avec les maillages utilisés.

- Il est très important de noter ici que nous avons arbitrairement déconnecté le traitement turbulent dans la zone film (aisé, car repérée par la valeur unitaire de la fonction de phase ou « couleur » unitaire) ; ceci sous l'argument qu'en raison des vitesses dans le film $\left(\mathrm{m} . \mathrm{s}^{-1}\right)$ et des épaisseurs observées $(10-100 \mu \mathrm{m})$ les nombres de Reynolds sont bas.

- L'équation d'Advection (3) permet de localiser l'interface au travers du transport de la variable $C$, «Couleur ou « Fonction de Phase », prenant les valeurs 1 en liquide et 0 dans le Gaz. La position de l'interface $(C=0,5)$ étant connue initialement, sa nouvelle position est calculée grâce à la connaissance du champ de vitesses, déterminé par la résolution de l'équation de Quantité de Mouvement. La résolution de cette équation doit être très précise : une équation de ce type hyperbolique a en effet tendance a être soit instable, soit fortement diffusive. Il a été développé, voir [7] encore, des méthodologies numériques alliant stabilité et diffusion numérique limitées, méthodes que nous employons ici. 


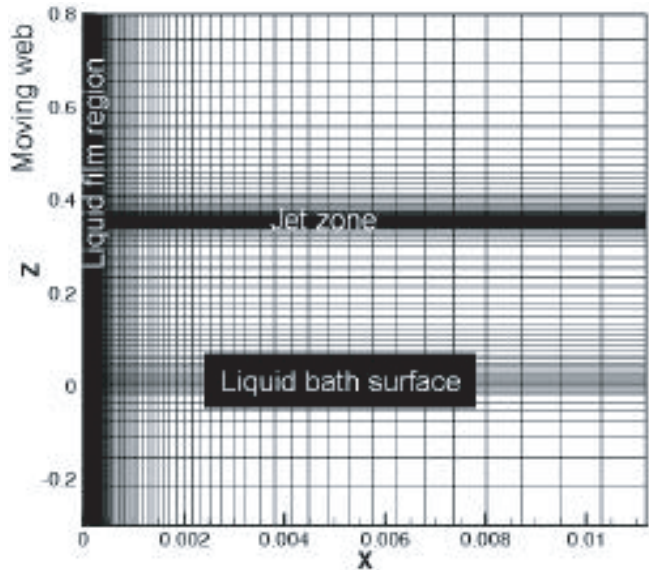

Fig. 2. Structuration typique du maillage.

- Enfin, le couplage Vitesse-Pression est résolu par l'utilisation de la technique du «lagrangien Augmenté ». Cette méthode consiste à intégrer la contrainte d'incompressibilité (autrement dit l'Éq. (2)) dans l'équation de quantité de mouvement [9]. Plus difficile à résoudre car on perd le caractère pentadiagonal du système à résoudre, cet algorithme est par contre très précis et efficace, voire indispensable pour résoudre proprement les problèmes d'écoulement avec interfaces sur maillage fixe.

Les équations précédentes sont discrétisées selon la méthode des Volumes Finis, sur un maillage fixe et cartésien structuré. On notera donc que le maillage ne s'adapte pas à la déformation de l'interface, mais que celle-ci traverse le maillage : c'est la valeur de la couleur dans une maille qui permettra de différencier liquide, gaz et zone d'interface $(C=0, C=1,0<C<1$, respectivement). Cette couleur permettra d'actualiser la valeur des caractéristiques thermophysiques locales (viscosité, masse volumique, conductivité thermique) et donc de différencier les zones fluides.

Le maillage est construit de manière à décrire correctement les échelles physiques des phénomènes : le jet est décrit par exemple dans sa largeur par 20 points (taille de maille verticale de l'ordre de $100 \mathrm{~mm}$ ), tandis que dans la direction de l'épaisseur du film 20 points décrivent le film essoré (typiquement d'épaisseur de 10 à $50 \mu \mathrm{m}$, soit une taille de maille horizontale de quelques microns), la couche limite d'impact du jet étant discrétisée par une vingtaine de points également. Cela conduit à des maillages typiquement de 100 selon $x$ par 200 selon $z$ distribués par zones (Fig. 2).

Même si l'on cherche ici généralement une solution stationnaire des phénomènes, le traitement de l'advection de matière (Éq. (3)) ainsi que la prise en compte de la turbulence par LES implique le recours à des pas de temps très petits (de $10^{-3} \mathrm{~s}$ pour le film remontant seul à $10^{-5} \mathrm{~s}$ pour le problème complet film + jet turbulent). Cette exigence amène à des temps de calculs significatifs, sans être cependant prohibitifs : pour un nombre de maille total de $200 \times 400$, le temps d'exécution sur un PC PentiumIV est de l'ordre de $72 \mathrm{~h}$, pour 200000 itérations, cf. temps physique d'établissement du film de l'ordre de 0,1 à $1 \mathrm{~s}$ et à l'obtention de moyennes temporelles représentatives.

L'outil numérique utilisé ici est un code généraliste de Mécanique des Fluides et de Transferts, AQUILON ${ }^{\mathrm{R}}$ développé au sein de notre Laboratoire TREFLE. Ce code a été validé par rapport à de nombreux problèmes académiques (convection naturelle, cavité entraînée, « backstep » problem, ...) ou industriels, notamment en rapport à la simulation des écoulements en présence d'interfaces (coulée, impacts de gouttes, ...) [http://www.trefle.u-bordeaux1.fr/aquilon].

\section{Résultats}

Le problème en considération fait intervenir différents sous-problèmes tout aussi riches les uns que les autres, qui peuvent être étudiés séparément :

- l'entraînement d'un liquide par une surface mobile (sans jet) ;

- l'interaction d'un jet (turbulent) avec une plaque sèche mobile.

Nous allons investiguer ces deux sous-problèmes avant de traiter le problème complet, ce qui nous permettra d'une part de valider notre procédure numérique mais également d'apprécier le rôle de certains paramètres opératoires.

\subsection{Développement d'un film entraîné par une plaque mobile}

\section{Analyse qualitative}

On peut voir sur la figure 3 la distribution des vecteurs vitesses à différentes hauteurs et au travers de l'interface liquide (rouge)-gaz (bleu). On note tout d'abord l'allure quasi-parabolique de la vitesse dans le film liquide, ce qui est théoriquement attendu (Poiseuille) et ensuite le changement de pente au passage de l'interface, ceci étant dû au contraste de viscosité : à l'interface, le profil de vitesse dans le liquide exhibe une pente quasi-nulle (analytiquement, on admet une contrainte de cisaillement nulle : on vérifie donc que cette hypothèse est justifiée). On remarque aussi que la couche limite dans le liquide est plus large dans la zone aérienne (b), (c), (d) que dans la zone bain (a) : ceci expliquera la présence d'un courant recirculant en pied de film, lorsque les deux couches limites se rencontrent.

Sur cette configuration on peut également se livrer à l'étude de l'effet de certains paramètres, par exemple la tension superficielle liquide-gaz. La comparaison de deux valeurs différant d'un facteur 10 par rapport à la valeur nominale de ce paramètre montre un effet sur le pied de film, où la courbure de l'interface se manifeste. Par contre, nous n'avons pas noté de différences notables sur l'épaisseur asymptotique de film. 


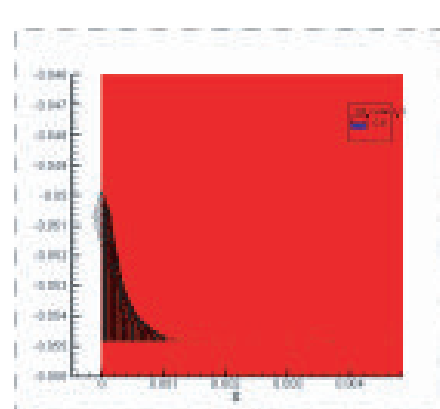

(a)

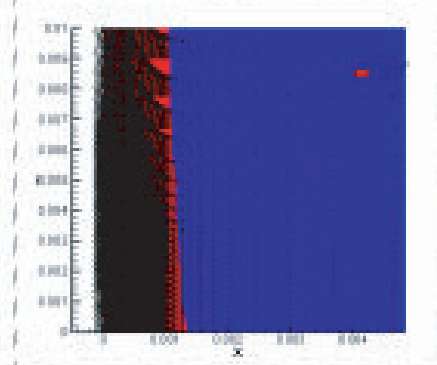

(b)

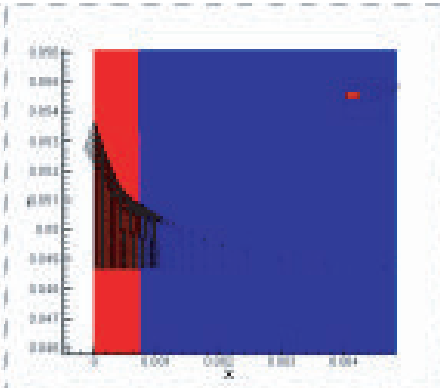

(c)

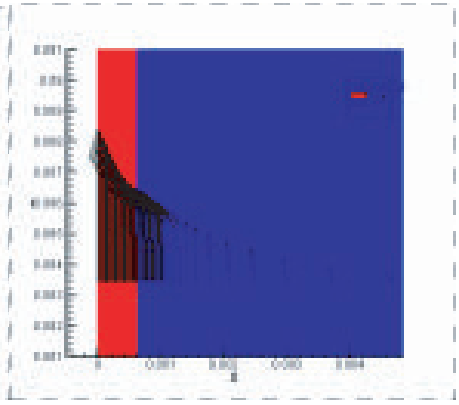

(d)

Fig. 3. Champ de vitesses à différentes hauteurs. (a) Dans le bain, (b) en zone de pied, (c) en zone intermédiaire, (d) en zone asymptotique.

\section{Analyse quantitative}

Une expression analytique de l'épaisseur asymptotique du film peut être établie pour ce problème, en invoquant évidemment des hypothèses simplificatrices. On montre ainsi que cette épaisseur varie pour un fluide donné comme la racine carrée de la vitesse de plaque :

$$
e_{\mathrm{film}}=k \sqrt{\mu V_{\mathrm{p}} / g}
$$

Sur la figure 4, on a reporté dans deux types de configurations académiques (a) et industrielles (b) les épaisseurs calculées par cette expression (5), par le modèle numérique et celles issues de mesures de la littérature déjà un peu anciennes [10]. Un accord est observé, même si les résultats numériques semblent s'éloigner de ceux de la théorie lorsque la vitesse de bande passe un certain seuil. Ce désaccord est à imputer au fait que l'inertie joue un rôle que ne prend pas en compte le modèle théorique.

\section{Étude d'un paramètre géométrique}

L'approche numérique permet de s'affranchir des hypothèses classiques à un modèle analytique. Nous avons par exemple pu tester l'effet de la hauteur du bain liquide, qui se confond ici avec la hauteur de plaque immergée (la plaque va jusqu'au fond du bain).

Conservant une hauteur émergée $H$ de $1 \mathrm{~m}$, nous allons faire varier la profondeur du bain $« h »$ de $1 \mathrm{~mm}$ jusqu'à $1 \mathrm{~m}$. Le résultat en terme d'épaisseur de film en haut de domaine ( $z=1 \mathrm{~m}$ au-dessus du bain) est résumé sur la figure $5 \mathrm{a}$ : on voit à l'évidence que la hauteur du bain joue un rôle déterminant sur la valeur de cette épaisseur. Pour des hauteurs faibles $(1 \mathrm{~mm}-1 \mathrm{~cm})$, on surestime notablement l'épaisseur asymptotique du film, celle-ci étant obtenue pour des hauteurs supérieures à $0,20-0,3 \mathrm{~m}$. Il est probable que cette hauteur critique soit d'autant plus forte que la vitesse de la plaque est elle-même élevée.

Pour analyser l'origine de cet effet de la hauteur de bain, il convient de comparer la topologie des écoulements en zone de pied de film. Pour une hauteur faible, le fluide qui alimente le film arrive en quelque sorte canalisé par la surface supérieure du bain (glissante) et le fond du bain (face adhérente) : les pertes de charge induites ne sont sûrement pas négligeables d'une part et

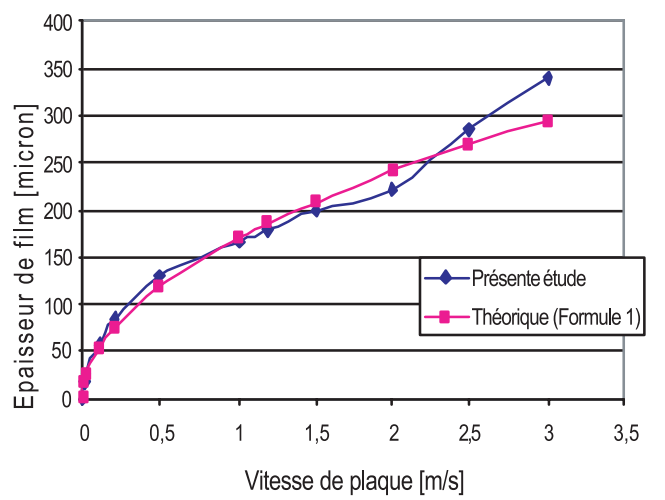

(a)

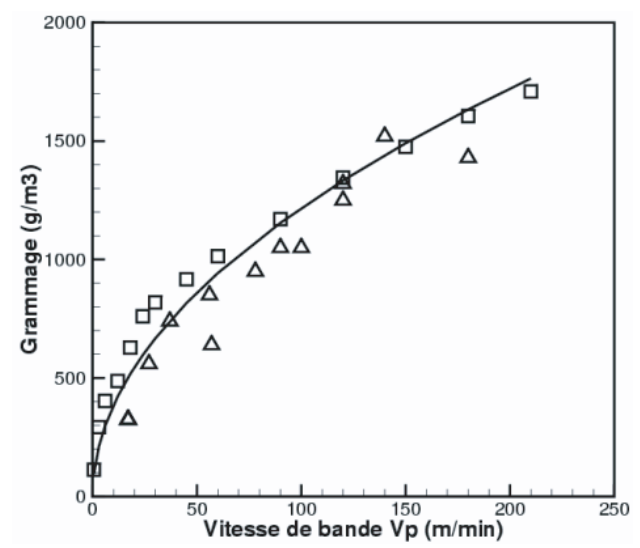

(b)

Fig. 4. Comparaison des épaisseurs (b) ou grammages de revêtement de métal liquide déposé par essorage gravitationnel mesurés, théoriques, et issus de la simulation ligne : valeurs théoriques $\triangle$ : données expérimentales [11], $\square$ : valeurs issues de la simulation Aquilon.

d'autre part la couche limite verticale sur la plaque entraînée a peu d'espace pour se développer. Il en résulte que le film est sous-alimenté par rapport à ce qu'il pourrait être si l'écoulement dans le bain n'était pas gêné et donc l'épaisseur du film est faible.

À l'inverse, lorsque la profondeur de bain est importante (Fig. 5b), la couche limite induite dans le bain par le 


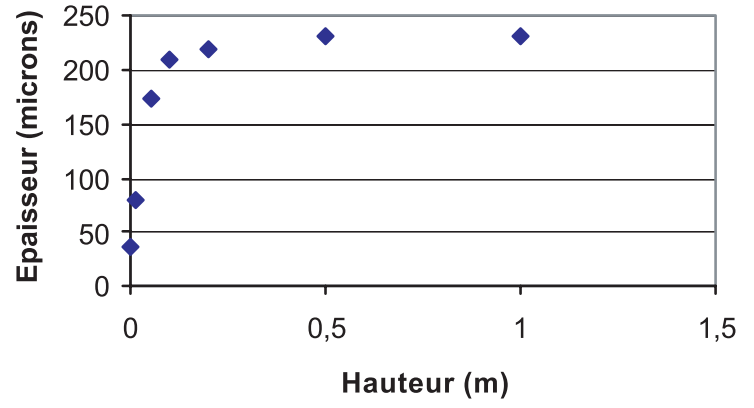

(a)

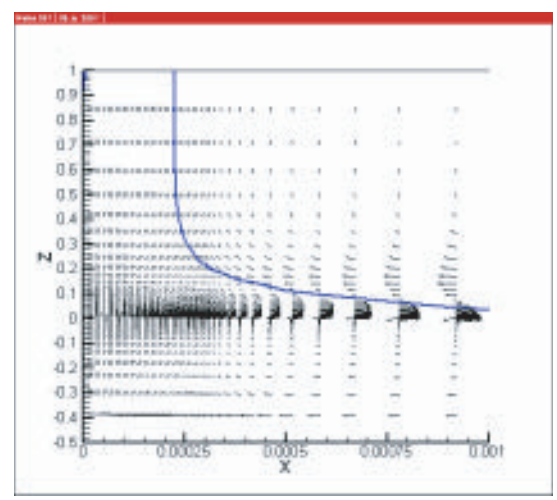

(b)

Fig. 5. (a) Variation de l'épaisseur du film en $z=1 \mathrm{~m}$ avec la hauteur du bain, (b) visualisation des écoulements en pied de film pour une profondeur de bain $(h)$ de $50 \mathrm{~cm}$.

glissement de la plaque se développe normalement, mais l'épaisseur de cette couche limite peut être plus grande que l'épaisseur de film entraîné dans la partie aérienne. Le résultat est un rebroussement de l'écoulement en pied de film, une partie, la plus proche de la plaque, étant effectivement entraînée vers le haut tandis qu'une autre partie retombe vers la surface libre du bain. Il en résulte un épaississement du film notamment en zone de pied (>1 mm contre $250 \mu \mathrm{m}$ précédemment).

\subsection{Interaction jet turbulent-plaque mobile}

Ce problème fait l'objet d'une bibliographie relativement abondante surtout lorsque la plaque est immobile [11-13]. Les paramètres essentiels sont le nombre de Reynolds de jet et le rapport de forme Distance Buse-Bande/Largeur de buse $(L / d)$. Dans les applications concernées, on se place dans une gamme de $L / d$ supérieure à 8 : c'est pour de telles valeurs que l'on peut estimer être au-delà du noyau potentiel du jet. En deçà, le jet devient très instable... et la simulation numérique très délicate. Nous avons qualifié notre outil numérique par rapport aux distributions de pression et de cisaillement sur la plaque, figure 6, soit par rapport aux références bibliographiques précitées : ce sont ces quantités qui évaluent les efforts transmis à la plaque (au film plus tard pour nous). Des modèles analytiques de prédiction d'essorage sont d'ailleurs basés sur ces distributions en supposant que la présence du film ne les affecte. Cette hypothèse est discutée plus amplement dans [14].

Les résultats numériques que nous avons obtenus dans les conditions qui nous intéressent (i.e. nombre de Reynolds de l'ordre de 10 et $L / d$ entre 5 et 12 ) sont en accord avec ceux de la littérature, mais il convient de noter que ceci nécessite un choix des paramètres du modèle LES (voir discussion en 2). Ce qui nous a conforté sur la validité de notre approche numérique est que la distribution de cisaillement mesurée au VKI sur une installation (Fig. 6b) est très bien reproduite (à condition de bien déterminer la localisation du dispositif de mesure...).

\subsection{Problème complet : l'essorage pneumatique}

Par rapport au problème de la montée du film présenté en section 3.1, nous introduisons maintenant l'effet d'un jet plan issu d'une buse en forme de fente de largeur « $d »$, frappant normalement la plaque défilante. La distance buse-plaque est dénommée $« L »$. En l'absence de plus d'informations, nous considérons qu'en sortie de buse le profil est uniforme, la vitesse étant $V_{\text {jet }}\left(200 \mathrm{~m} . \mathrm{s}^{-1}\right)$. Les paramètres géométriques du jet et la vitesse sont choisis de telle sorte qu'un effet notable sur le film est produit.

\section{Analyse de la déformation de l'interface}

La forme du film dans la région d'impact du jet $(z=0)$ est représentée très dilatée sur la figure 7 : on voit que l'épaisseur passe typiquement de plusieurs centaines de microns à une vingtaine de microns. On voit même le re-épaississement (léger) du film lié au rétablissement du profil de vitesse lorsque l'effet du cisaillement s'estompe. Le modèle analytique et la simulation sont assez en accord : par contre le modèle analytique surestime les épaisseurs de film redescendant et surtout ne permet pas de reproduire des phénomènes d'instabilités tels que le splashing que nous allons étudier après. Nous avons comparé les épaisseurs asymptotiques calculées aux épaisseurs mesurées au VKI sur une maquette « eau » en faisant varier la distance buse-bande dans une large gamme (Fig. 8). On voit que simulation et expérience relèvent un changement de comportement pour une valeur de $L / d$ entre 6 et 8 ce qui correspond à l'extension du noyau potentiel. En deçà, la sensibilité à ce paramètre semble moindre. Les résultats pour de faibles valeurs de $L / d$ ne sont plus autant en accord : nous reconnaissons que le calcul numérique devient très instable et nous pensons que les effets de compressibilité deviennent alors significatifs.

\section{Analyse du phénomène de «splashing »}

La méthode numérique de suivi d'interface que nous utilisons peut prendre en compte des ruptures de film liquide, donc la création de gouttes (Fig. 9), et leur éventuelle coalescence. Nous utilisons cette fonctionnalité pour reproduire le phénomène observé sur les installations industrielles : au-delà d'une certaine valeur de la 

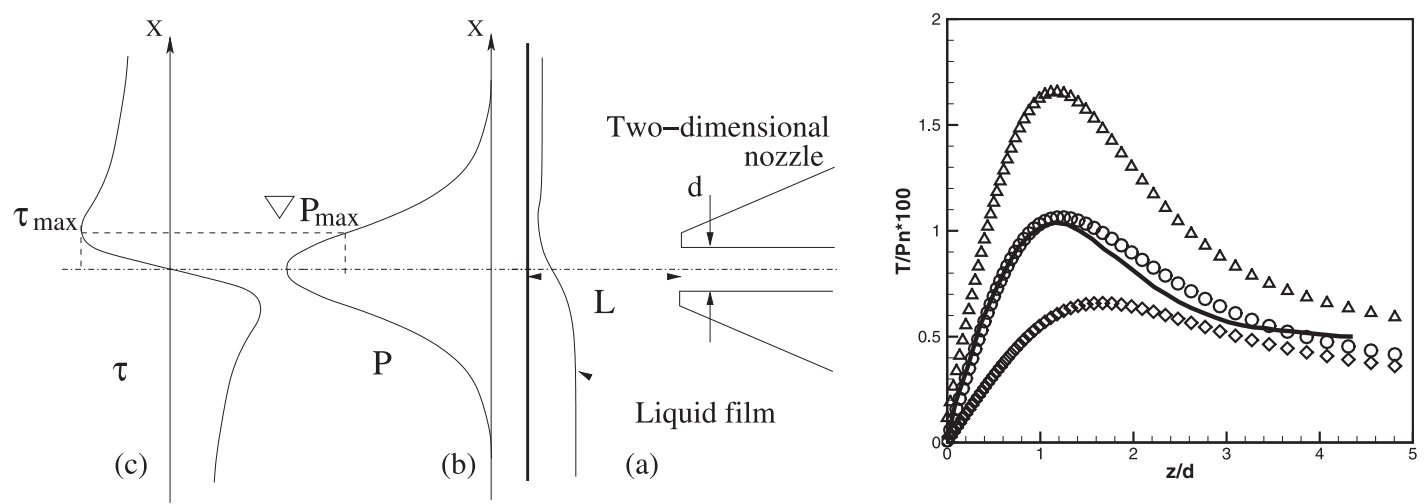

Fig. 6. (a) Visualisation symbolique des distributions de pression et de cisaillement lors de l'impact d'un jet sur plaque sèche immobile, (b) comparaison entre distributions calculées à différents endroits (symboles) et mesurée (ligne continue) au VKI [14].

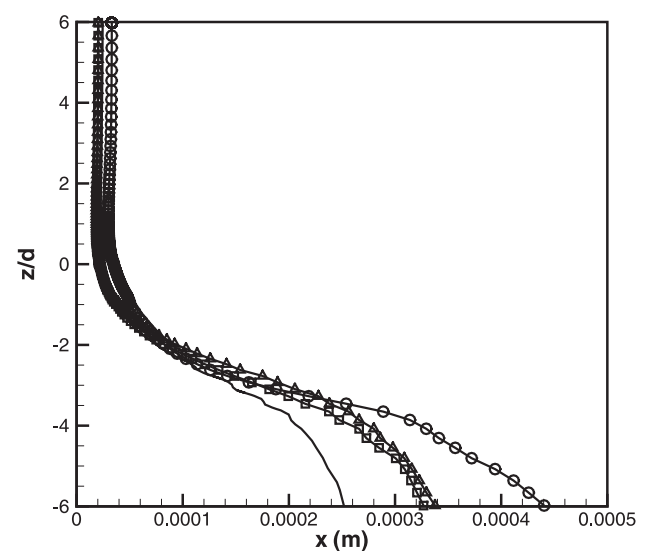

Fig. 7. Forme de l'interface (zoom dans la zone d'impact du jet), comparaison entre modèle analytique avec différentes distributions de pression-cisaillement et simulation (ligne).

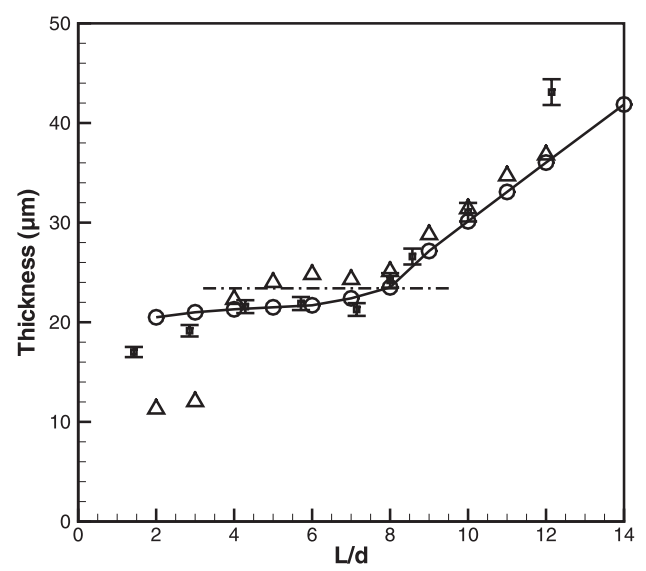

Fig. 8. Effet de la distance buse-bande $(L)$ normalisée par la largeur de buse $(d)$ sur l'épaisseur asymptotique (triangle : simulation - carré : expérimental VKI - rond : analytique calé).

vitesse de plaque, apparaît un «splashing » autrement dit des éclaboussures. Bien que notre modélisation numérique soit assez imparfaite (la plus grande critique venant de la réduction $2 \mathrm{D}$ et dans une moindre mesure l'approximation de tension superficielle constante alors que les gra-

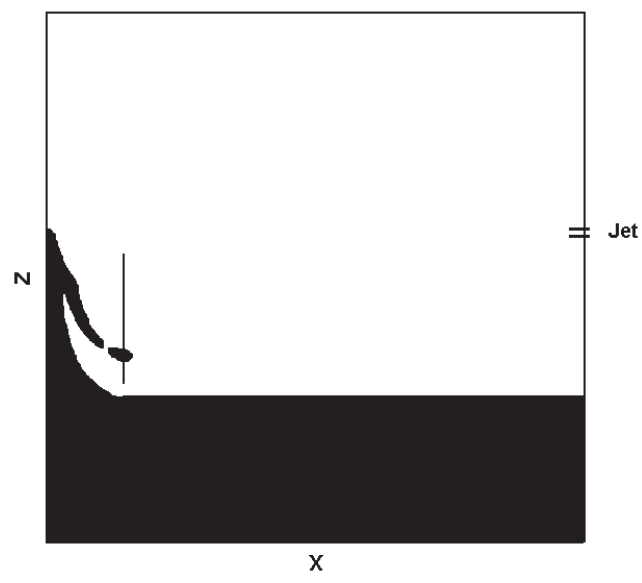

Fig. 9. Visualisation du splashing (la ligne verticale figure le « capteur $»$ permettant d'identifier l'apparition du phénomène).

dients de température sont élévés), la comparaison du diagramme de stabilité expérimental obtenu sur une maquette eau à l'IVK [15] et nos prédictions numériques sont en bon accord (Fig. 10). On constate aussi bien pour l'eau que pour le zinc que la vitesse de bande est plus déterminante encore que la vitesse du jet. En effet, à quelques écarts près à haute et faible vitesse de jet, quelle que soit cette vitesse du jet, le splashing apparaît toujours autour d'une même valeur de la vitesse de bande. La vitesse de bande étant le principal paramètre contrôlant l'épaisseur avant essorage, on peut postuler que les gouttelettes se décrochent lorsque la quantité de liquide entraînée est trop importante, c'est-à-dire lorsque l'épaisseur est supérieure à une valeur qu'on pourrait appeler critique. De plus, un autre critère d'apparition, qui ressort de l'examen des différentes simulations que nous avons menées, est l'angle formé par le repli du zinc sous l'essorage (à vrai dire ce n'est peut-être pas un autre critère, la forme du repli étant elle-même une conséquence des interactions film-jet) : dès que l'angle formé avec la verticale est trop grand, le splashing apparaît. De la même manière, plus l'épaisseur est importante, plus l'angle est grand. On peut penser que le jet heurtant le repli du film 


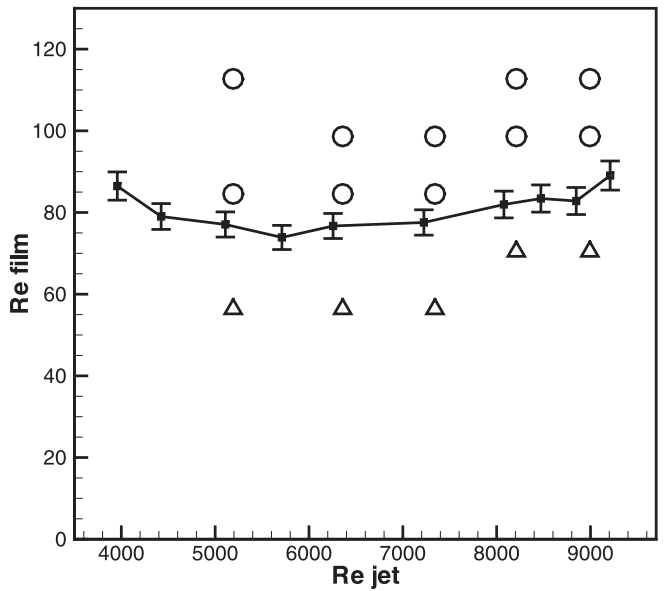

Fig. 10. Diagramme d'apparition du splashing en fonction de la vitesse de jet et de la vitesse de plaque (symboles : points calculés, ligne : limite de stabilité par repérage expérimental visuel).

de zinc de manière plus directe, il favorise une instabilité de surface qui conduit au décrochement des gouttes.

\section{Conclusions-perspectives}

Nous avons montré dans cet article que la simulation numérique directe du couplage hydrodynamique entre deux phases non miscibles (liquide-gaz) permettait de reproduire et d'analyser le phénomène d'entraînement d'un fluide par une plaque et de son amincissement sous l'effet d'un jet turbulent. Des effets plus locaux comme le splashing (arrachage de gouttes par le jet) sont également rendus.

La simulation LES a montré sa possibilité à être couplée à un traitement d'interface et donc d'un problème diphasique. Si nos résultats semblent corroborés par des expérimentations extérieures en terme d'épaisseur de film ou d'instabilités de film, il n'en reste pas moins que ceci a été au prix de choix arbitraires en terme de modélisation de la turbulence. Des simulations numériques 3D récentes, qui méritent d'être complétées, montrent en effet que les épaisseurs de film observées expérimentalement sont reproduites numériquement à condition de prendre une définition standard du filtre spatial (relation 4) et surtout de ne pas déconnecter la procédure LES dans la zone film comme nous l'avons fait ici dans l'approche 2D. Cela fait pressentir une transmission partielle de la turbulence du jet vers le film, mais il convient d'approfondir cette idée.

Pour être plus près des conditions industrielles, il conviendrait enfin de tenir tout d'abord compte du caractère (partiellement) compressible de l'écoulement de jet en raison du nombre de Mach assez fort, et également de prendre en compte le couplage thermique, important a priori au travers de ses effets sur la masse volumique et la tension superficielle.

Remerciements. Les auteurs adressent leurs remerciements à l'IDRIS et au CINES (Projet No. TER2237-2004) pour leur soutien sous forme d'heures de calcul. Ils remercient aussi le Professeur J.M. Buchlin du VKI de Bruxelles pour les discussions fructueuses et les résultats expérimentaux communiqués.

\section{Références}

[1] C.H. Ellen, C.V. Tu, An analysis of jet stripping of liquid coatings, J. Fluids Eng. 106 (1984) 399

[2] L. Landau, B. Levich, Dragging of a liquid by a moving plate, Acta Physica USSR 17 (1942) 42-54

[3] J.A. Thornton, M.F. Graff, An analytical description of the jet finishing process for hot-dip metallic coating on strip, Metall. Trans. B 7B (1976) 607

[4] J.-M. Buchlin, Modeling of gas-jet wiping, Thin Liquid Films and Coating Processes, VKI Lecture Series, von Karman Institute for Fluid Dynamics, 1997

[5] J.U. Brackbill, B.D. Kothe, C. Zemach, A continuum method for modelling surface tension, J. Comput. Phys. 100 (1992) 335

[6] R. Scardovelli, S. Zaleski, Direct numerical simulation of free-surface and interfacial flow, Annu. Rev. Fluid Mech. 31 (1999) 567

[7] S. Vincent, J.-P. Caltagirone, Efficient solving method for unsteady incompressible interfacial flow problems, Int. J. Numer. Methods Fluids 30 (1999) 795

[8] P. Sagaut, Large Eddy Simulation for Incompressible Flows, Springer-Verlag, Berlin, 1998

[9] S. Vincent, J.-P. Caltagirone, P. Lubin, T.N. Randrianarivelo, An adaptive augmented Lagrangian method for three-dimensional multimaterial flows, Comput. Fluids 33 (2004) 1273

[10] A. Nikoleizig, T. Kootz, F. Weber, M. Espenhahn, Fundamentals of the Jet Process for Hot Dip Galvanising, Stahl und Eisen 98 (1978) 336

[11] C.V. Tu, D.H. Wood, Measurements beneath an impinging plane jet, Exp. Therm. Fluid Sci. 13 (1996) 364

[12] D.J. Phares, G.T. Smedley, R.C. Flagan, The wall shear stress produced by the normal impingement of a jet on a flat surface, J. Fluid Mech. 418 (2000) 351

[13] S. Maurel, C. Solliec, A turbulent plane jet impinging nearby and far from a flat plate, Exp. Fluids 31 (2001) 687

[14] D. Lacanette, A. Gosset, S. Vincent, J.M. Buchlin, E. Arquis, Macroscopic Analysis og gas-jet wiping : numerical simulation and experimental approach, J. Phys. Fluids 18 (2006) 042103

[15] D. Lacanette, S. Vincent, E. Arquis, P. Gardin, Numerical simulation of gas-jet wiping in steel strip galvanizing process, ISIJ Int. 45 (2005) 2142 